\title{
Anisotropic Deformation Behavior of Al2024T351 Aluminum Alloy
}

\author{
R Khan \\ Mechanical Engineering Department, NED University of Engineering and Technology, Karachi, Pakistan
}

Received 15 October 2011 ; accepted 28 July 2012

\begin{abstract}
The objective of this work was to investigate the effects of material anisotropy on the yielding and hardening behavior of 2024T351 aluminum alloy using isotropic and anisotropic yield criteria. Anisotropy may be induced in a material during the manufacturing through processes like rolling or forging. This induced anisotropy gives rise to the concept of orientation-dependent material properties such as yield strength, ductility, strain hardening, fracture strength, or fatigue resistance. Inclusion of the effects of anisotropy is essential in correctly predicting the deformation behavior of a material. In this study, uniaxial tensile tests were first performed in all three rolling directions, $L, T$ and $S$, for smooth bar specimens made from hot rolled plate of A12024 alloy. The experimental results showed that the $L$ - and $T$-directions yielded higher yield strengths and a greater percentage of elongation before fracture than the $S$-direction. Subsequently, finite element analysis of tensile specimens was performed using isotropic (von Mises) and anisotropic (Hill) yield criteria to predict the onset of yielding and hardening behaviors during the course of deformation. Hill's criterion perfectly fitted with the test data in the $S$-direction, but slightly underestimated the yield strength in $L$-direction. The results indicated that the Hill yield criterion is the most suitable one to predict the onset of yielding and hardening behaviors for $2024 \mathrm{~T} 351$ aluminum alloy in all directions.
\end{abstract}

Keywords: Deformation behaviour, Anisotropy, Downhole expandable tubular, Finite element analysis

$$
\begin{aligned}
& \text { سلوك التثوه المتباين الخواص لسبائك الألومنيوم Al2024T351 } \\
& \text { راشد خان }
\end{aligned}
$$

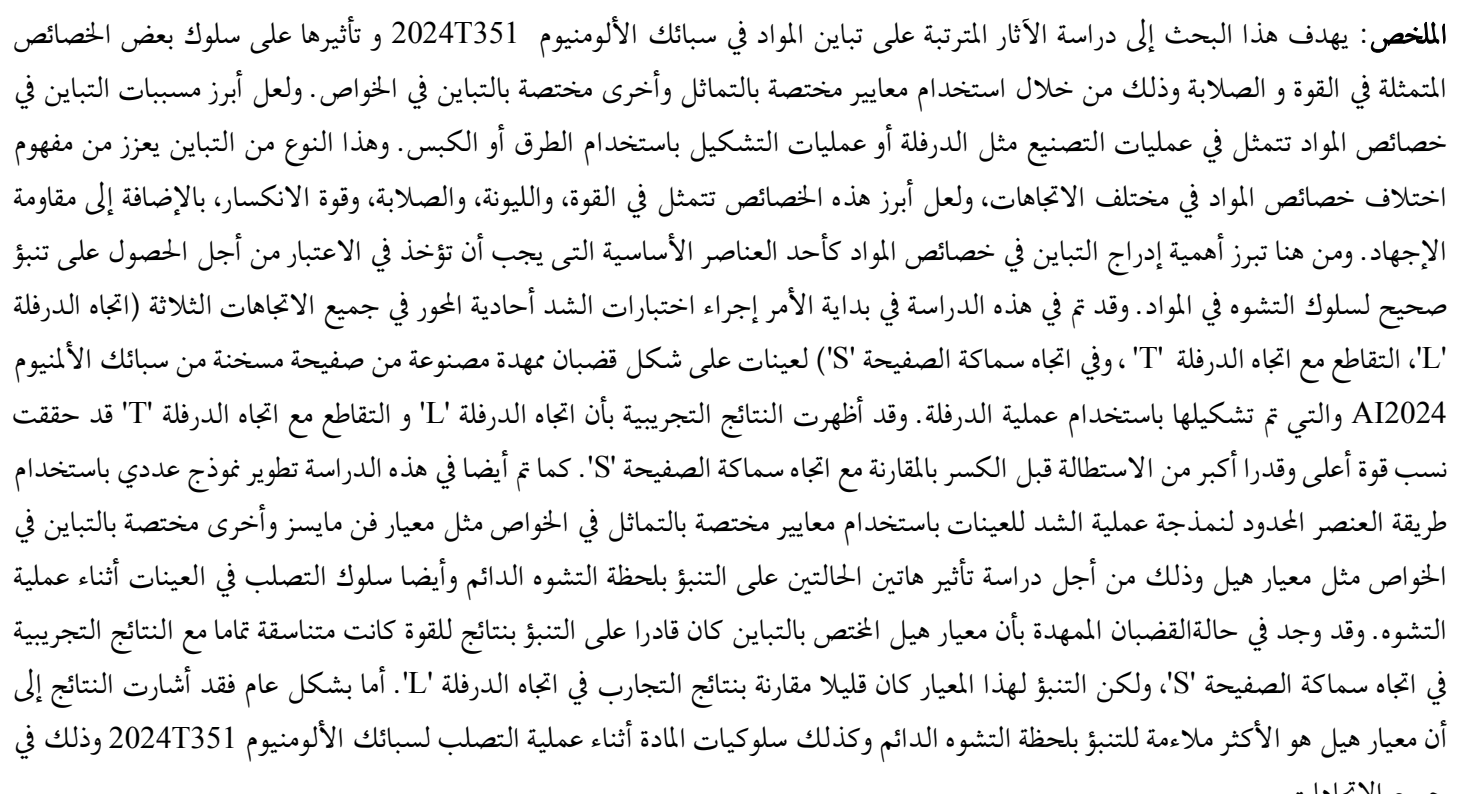

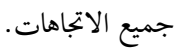
المفردات المفتاحية: سلوك التشوه، تباين الخواص، تمديد الأنابيب لتطبيقات الحفر، التحليل بطريقة العنصر الخدود.

*Corresponding author's e-mail: rashidkh@neduet.edu.pk 


\section{Introduction}

Aluminum alloys are well-known for their excellent mechanical properties such as a high strength-toweight ratio, resistance to corrosion, and strength integrity at low temperatures. These properties have led to its extensive use in aerospace and cryogenic applications. For decades, thin rolled sheets of aluminum alloy 2024T351 have been used for aircraft applications (Starke 1996; Nakai 2000). Like other aluminum alloys, the 2024 alloy exhibits anisotropy in its deformation and fracture behaviors. Although extensive experimental work, along with analytical and numerical analyses, has been performed (Lam 2010; Lee 1999; Mimaroglu 2003; Schmitt 1998) for predicting the isotropic and anisotropic deformation behaviors of aluminum 2024 alloys, so far its deformation-induced anisotropy has not been completely understood. Anisotropy is induced in a material during manufacturing through processes like rolling or forging. The induced anisotropy results in directionaldependent material properties such as yield strength, ductility, strain hardening, fracture strength, and fatigue resistance. Numerous studies have been done to evaluate the material properties of 2024 aluminum alloys (Bron 2006, 2004b; Srivatsan 2007; Shanmuga 2010). A thorough understanding of the effects of induced anisotropy is essential to correctly predicting the deformation behavior of a material. In thin plates made of 2024 alloy, the properties are strongly dependent on the rolling direction (Steglich 2008), while the other two orientations (ie. thickness and width) are not critical in predicting the deformation behavior. However, for thick plates the anisotropy along thickness and width directions cannot be neglected in estimating the deformation behavior under different loading conditions. The onset of yielding, the progression of plastic deformation, and work hardening are not the same in all directions.

Researchers have carried out numerous studies and developed various yield criteria, plastic flow rules, and work/strain hardening hypotheses to predict deformation-induced anisotropy for ferrous alloys. These same criteria, rules, and hypotheses are assumed to be equally applicable for aluminum alloys with some modifications. However, the yield criterion which is most applicable for 2024 alloy for all directions, and which agrees well with experimental results, remains a topic of research. There is no reported literature on this subject and filling this void is the main objective of the current study.

Downhole tubular expansion technology in the oil and gas industry has demonstrated its enormous influence on the overall drilling cost of wells (Campo 2003). Many issues remain unresolved in spite of its successful implementation. One of these issues is related to induced anisotropy in downhole tubular material during the cold expansion process. In the expansion process, the tube might not become deformed uniformly due to the presence of non-homogeneities as a result of the manufacturing process. The non-uniform expansion process results in the anisotropic behavior of downhole tubes and ultimately affects their structural integrity. Therefore, a careful analysis of induced anisotropy is necessary to estimate its effects on the structural integrity of downhole tubes. This work focused on analyzing an induced anisotropy in a material during deformation, while the relation between an induced anisotropy in a downhole tube with its structural integrity will be included in future work. Also, this research is an addition to the work reported by Pervez (2008), where the postexpansion properties of downhole tubular materials like expansion force, thickness reduction, length shortening, and contact pressure were compared in two different types of materials steel and aluminum. The authors concluded that aluminum is a better option for downhole tubular construction due to its lower energy requirement for expansion, high formability, and good corrosion resistance. This work contains the effects of anisotropy on the deformation behavior of downhole tubular materials, while assuming that the downhole tube is made up of A12024 aluminum alloy.

A law defining the onset of yield under a combined state of stress is known as yield criterion. The generalized von Mises yield criterion for isotropic materials (1913) accurately predicts the onset of yielding for most polycrystalline metals as compared to the criterion of (Tresca 1864). An extension of von Mises's yield function was first proposed by Hershey (1954), and then was generalized by Hosford (1972). The loci of yield surface of isotropic yield function by Hosford lies between that proposed by von Mises and Tresca. The equivalent stress by Hosford is given as:

$$
\sigma_{e}=\left[\frac{1}{2}\left(\left|\sigma_{2}-\sigma_{3}\right|^{a}+\left|\sigma_{3}-\sigma_{1}\right|^{a}+\left|\sigma_{1}-\sigma_{2}\right|^{a}\right)\right]^{1 / a}
$$

where $\sigma_{i, i=1,2,3}$ are the principal values of deviatoric stress $\overline{\bar{\sigma}}_{d}$, while $a$ is the material parameter. The Hosford yield function reduces to von Mises when $a$ equals 2 and to Tresca's yield function when $a$ equals 1. The material parameter $a$ is dependent on crystallographic structure (Logan 1980; Hosford 1996). The value of $a$ is 6 and 8 for body centred 
cubic (BCC) and face centred cubic (FCC) crystals, respectively. The first anisotropic yield criterion was proposed by Hill (1950) for materials having orthotropic symmetry, such as rolled sheets of metals. The effects of anisotropy in Hill's yield criterion are described by six parameters: $q_{i, i=1-6}$. The equivalent stress is defined as

$$
\sigma_{e}=\left[\begin{array}{l}
q_{1}\left(\sigma_{22}-\sigma_{33}\right)^{2}+q_{2}\left(\sigma_{33}-\sigma_{11}\right)^{2} \\
+q_{3}\left(\sigma_{11}-\sigma_{22}\right)^{2}+2 q_{4} \sigma_{23}^{2}+2 q_{5} \sigma_{31}^{2} \\
+2 q_{6} \sigma_{12}^{2}
\end{array}\right]_{(2)}^{1 / 2}
$$

where $\sigma_{i j, i, j=1,2,3}$ denotes the components of stress tensor $\overline{\bar{\sigma}}$. The anisotropic parameter $q_{i}$ is determined using Hill's stress ratio: $R_{i j, i, j=1,2,3}$.

$$
\begin{aligned}
& q_{1}=\frac{1}{2}\left(\frac{1}{R_{22}^{2}}+\frac{1}{R_{33}^{2}}-\frac{1}{R_{11}^{2}}\right), \\
& q_{2}=\frac{1}{2}\left(\frac{1}{R_{33}^{2}}+\frac{1}{R_{11}^{2}}-\frac{1}{R_{22}^{2}}\right), \\
& q_{3}=\frac{1}{2}\left(\frac{1}{R_{11}^{2}}+\frac{1}{R_{22}^{2}}-\frac{1}{R_{33}^{2}}\right), \\
& q_{4}=\frac{3}{2 R_{23}^{2}}, \quad q_{5}=\frac{3}{2 R_{13}^{2}}, q_{6}=\frac{3}{2 R_{12}^{2}},
\end{aligned}
$$

where, $R_{i j}$ is defined as

$$
\begin{aligned}
& R_{11}=\frac{\sigma_{11}}{\sigma_{y}}, R_{22}=\frac{\sigma_{22}}{\sigma_{y}}, R_{33}=\frac{\sigma_{33}}{\sigma_{y}}, \\
& R_{12}=\frac{\sigma_{12}}{\tau_{y}}, R_{23}=\frac{\sigma_{23}}{\tau_{y}}, R_{13}=\frac{\sigma_{13}}{\tau_{y}},
\end{aligned}
$$

and $\sigma_{y}$ and $\tau_{y}$ are the yield and shear strengths of a material. The yield function given by Eqn. (2) is widely used for steels. However, it has been found to be less accurate in the case of aluminum alloys; attempts have been made to find an appropriate yield criterion for aluminum alloys. Another extension of isotropic yield function, Eqn. (1), for anisotropic materials was proposed by Barlat (1991) where a yield criterion was proposed consisting of a modified deviatoric stress tensor $\overline{\bar{\sigma}}_{d}^{m}$ instead of $\overline{\bar{\sigma}}_{d}$. The modified deviatoric stress tensor is expressed as

$$
\overline{\bar{\sigma}}_{d}^{m}=\overline{\overline{\bar{L}}}: \overline{\bar{\sigma}}
$$

where $\overline{\bar{L}}$ is the fourth order tensor which incorporates the anisotropic effects in the yield function. However, (Karafillis 1993) showed that $\overline{\overline{\bar{L}}}$ has strong orthotropic symmetry and can be reduced to the sixth anisotropic parameter. Many attempts have been made to improve $\overline{\bar{L}}$ by introducing additional anisotropic coefficients (Karafillis 1993; Barlat 1997a). Recently, (Bron 2004a) proposed an advanced and modified form of Karafillis's yield function (1993). The equivalent stress is given by

$$
\sigma_{e}=\left[\sum_{k=1}^{K} \alpha^{k}\left(\overline{\bar{\sigma}}^{k}\right)^{a}\right]^{1 / a}
$$

where $K$ function $\overline{\bar{\sigma}}^{k}$ is the deviatoric stress function, $a$ is the material parameter, and $\alpha^{k}$ are positive coefficients such that $\sum_{k=1}^{K} \alpha^{k}=1$. The stress for two functions $(K=2)$ in Eqn. (4) is defined as follows:

$$
\begin{aligned}
& \overline{\bar{\sigma}}^{k}=\left(\varphi^{k}\right)^{1 / b^{k}} \\
& \varphi^{1}=\frac{1}{2}\left(\begin{array}{c}
\left|\sigma_{2}^{1}-\sigma_{3}^{1}\right|^{b^{1}}+\left|\sigma_{3}^{1}-\sigma_{1}^{1}\right|^{b^{1}} \\
+\left|\sigma_{1}^{1}-\sigma_{2}^{1}\right|^{b^{1}}
\end{array}\right) \\
& \varphi^{2}=\frac{3^{b^{2}}}{2^{b^{2}}+2}\left(\begin{array}{c}
\left|\sigma_{1}^{2}\right|^{b^{2}}+\left|\sigma_{2}^{2}\right|^{b^{2}} \\
+\left|\sigma_{3}^{2}\right|^{b^{2}}
\end{array}\right)
\end{aligned}
$$

where $\sigma_{i}^{k}, i=1,2,3, k=1,2$ are the principal values of $K$ - function's deviatoric stress tensor, $\overline{\bar{\sigma}}_{d}^{k}$. The $K$-functions of deviatoric stress is defined in a similar manner to Eqn. (3), as

$$
\overline{\bar{\sigma}}_{d}^{k}=\overline{\overline{\bar{L}^{k}}}: \overline{\bar{\sigma}}
$$




$$
\overline{\overline{\bar{L}}}^{k}=\left[\begin{array}{cccccc}
\left(c_{2}^{k}+c_{3}^{k}\right) / 3 & -c_{3}^{k} / 3 & -c_{2}^{k} / 3 & 0 & 0 & 0 \\
-c_{3}^{k} / 3 & \left(c_{3}^{k}+c_{1}^{k}\right) / 3 & -c_{1}^{k} / 3 & 0 & 0 & 0 \\
-c_{2}^{k} / 3 & -c_{1}^{k} / 3 & \left(c_{1}^{k}+c_{2}^{k}\right) / 3 & 0 & 0 & 0 \\
0 & 0 & 0 & c_{4}^{k} & 0 & 0 \\
0 & 0 & 0 & 0 & c_{5}^{k} & 0 \\
0 & 0 & 0 & 0 & 0 & c_{6}^{k}
\end{array}\right]
$$

The constants $a, b^{1}, b^{2}$, and $\alpha=\alpha^{1}$ are material parameters which control the yield surface, but the induced anisotropy is only controlled by $c_{i}^{k}, \quad i=1-6, k=1,2$. Hence, the proposed yield function by Bron (2004a) involves 16 material parameters. For the conditions $\overline{\overline{\bar{L}}}=\overline{\overline{\bar{L}}}, a=b^{l}=b^{2}$, and $\alpha=1$, the Bron yield function reduces to Karafillis's yield function. Similarly, for $\alpha=1$ and $c_{i}^{1}=1$, Bron yield function reduces to von Mises when $b^{l}=2$ and to Tresca's when $b^{l}=1$. Finally, for $c_{i}^{k}=1$, the yield function reduces to isotropic condition. The proposed yield function by Bron consists of a general formulation, which makes it possible to be extended easily by adding new $K$ functions in the summation; defining equivalent stress (Eqn. 4). The proposed yield function by Bron has been found very accurate in describing the plastic anisotropy of various aluminum sheet samples.

The current work is aimed at the prediction of yielding and hardening behaviors, resulting from different loading conditions and specimen geometries for the Al2024T351 alloy using different yield criteria. Uniaxial tensile tests were performed on smooth bar specimens. FE simulations were done in FE code Abaqus for smooth bar specimen using different yield criteria to find out which single yield criterion could be used to fit the hardening curve in all three directions. The numerical results were validated using experimental data.

\section{Experimental Study}

Uniaxial tensile tests were performed on smooth bar specimens made from a hot rolled plate of 2024 alloy in $L-, T$ - and $S$-directions. The material orientations with respect to rolling and loading directions are shown in Figures 1a and b. Before cutting the sample, the plate was solution heat treated, air quenched, and stress relieved by cold stretching. All samples had a uniform thickness of $100 \mathrm{~mm}$. The gauge length of the smooth bar specimen was $50 \mathrm{~mm}$ with a diameter of $10 \mathrm{~mm}$. The main alloying element in 2XXX series of aluminum alloy was copper, while magnesium and manganese were added to improve the quenching properties. The composition of the Al2024 alloy is given in Table 1.

\section{Finite Element Analysis}

Further to experimental work, the finite element analysis for predicting the deformation behavior of the A12024T351 alloy in different rolling directions was performed for both smooth and notched bars specimens using commercial finite element code ABAQUS with different yield criteria. Twodimensional (2-D) and three-d imensional (3-D) finite element models were developed using C3D8R elements. An elastic-plastic material model was used with isotropic hardening. The stress-strain data points were obtained from experimental results, which were used to define the constitutive relation in FE analysis. Material parameters included the yield strength in the $S$-direction ( $\sigma_{Y}^{S}=344 \mathrm{MPa}$ ), yield strength in the $L$ direction $\left(\sigma_{Y}^{L}=395 \mathrm{MPa}\right)$, Young's modulus ( $E=$ $700 \mathrm{GPa})$, and Poisson's ratio $(v=0.33)$. The isotropic yield criterion of von Mises was implemented in the material model by taking all stress ratios as given in Eqn. (2b) equal to one. Hill's yield criterion was used in the material's definition by defining the stress ratios given in Eqn. (2b).

\section{Results and Discussions}

The experimental results for the uniaxial tensile tests of smooth bar are shown in Fig. 2. For smooth bar, the yield point remained the same in $L$ - and $T$ directions, but was of a lower value in the S-direction. For smooth bar, the percentage elongations in $L, T$ and $S$-directions were 22, 19.6, and 9, respectively. This 
Table 1. Composition of 2024 aluminium alloy by weight percent (\%)

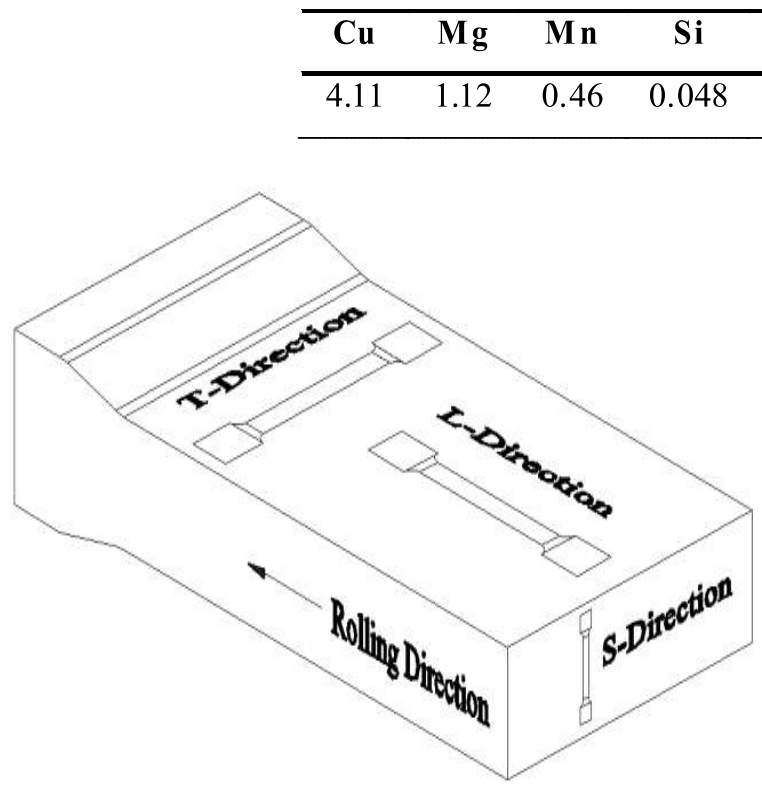

(a)

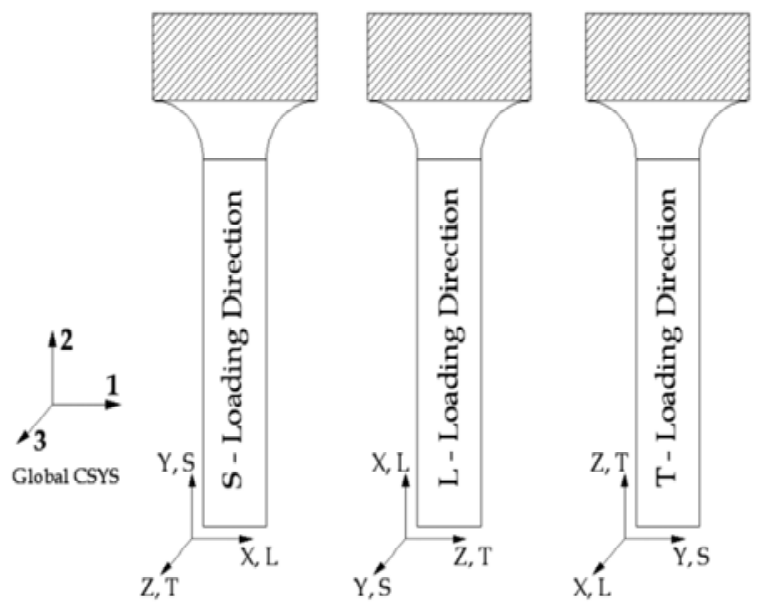

(b)

Figure 1. (a) Thin rolled plate of aluminium 2024T351 alloy; (b) Material orientation with respect to loading directions $(1,2,3)$ and $(x, y, z)$ are global and local coordinate systems, respectively

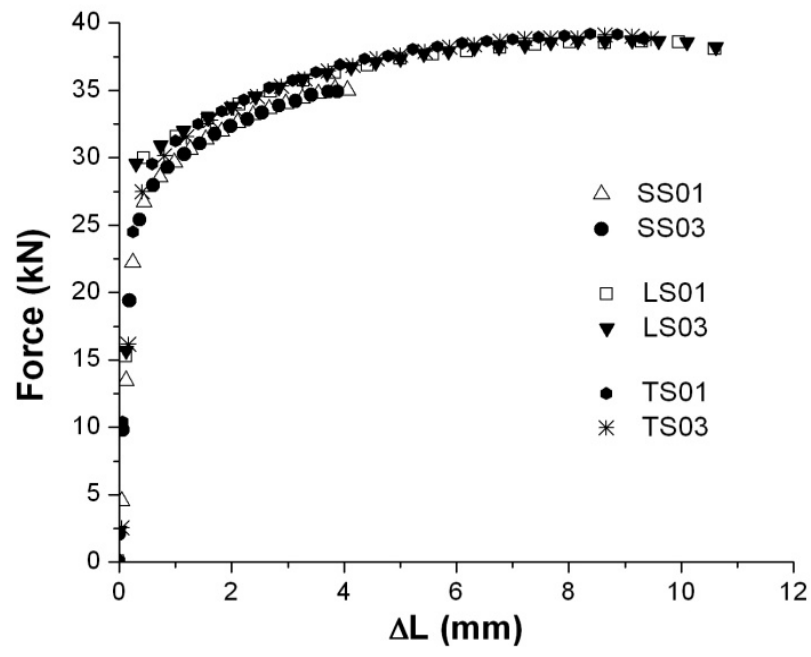

Figure 2. Experimental results of tensile tests for smooth bar specimens, SS01 has an $S$-direction smooth bar in Experiment 1 and SS03 has an $S$-direction smooth bar in Experiment 3. The findings are similar in the $L$ - and $T$-directions)

means that the material exhibited less ductility in the thickness direction as compared to the rolling and width directions.

\subsection{Smooth Bar Specimen}

The finite element analyses for smooth bar specimens were performed using isotropic and anisotropic deformation behaviors. Figure 3 shows that the uniaxial tensile test data of two speci- smens for the $S$-direction agreed well with the 2-D finite element results. Also, the 2-D and 3-D finite element results are compared in Fig. 3(b) indicating that the 2-D model was sufficient in this case. It is important to note that this result was used as a reference for the $L$-direction. For anisotropic deformation behavior, Hill's yield criterion was used for both $S$ - and $L$-directions. It can be observed from experimental results that the yield and ultimate 


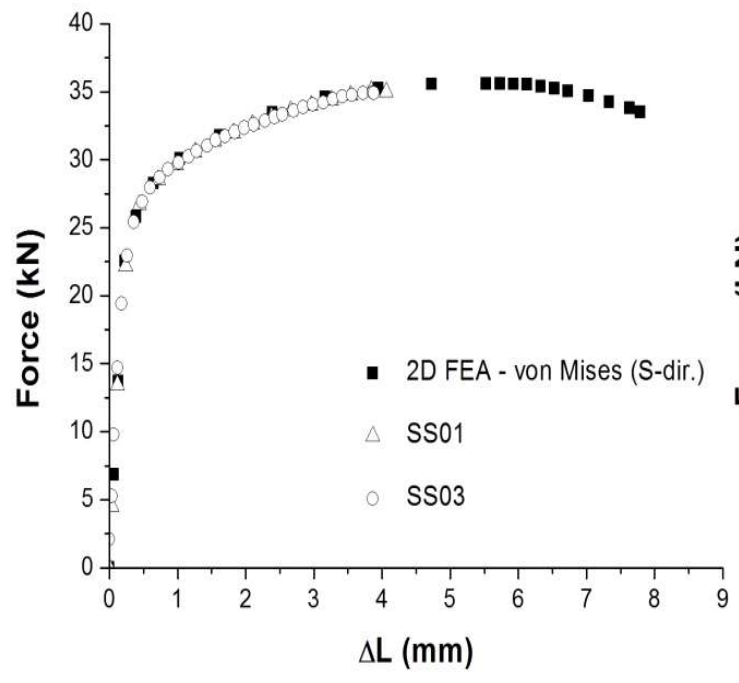

(a)

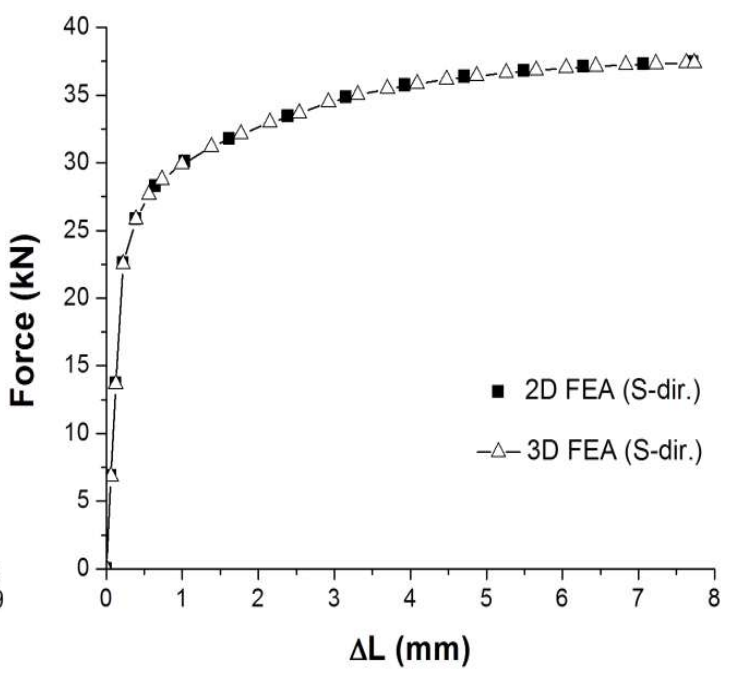

(b)

Figure 3. Force versus elongation in the S-direction using von Mises yield criterion; (a) 2-D finite element analyses (FEA); (b) 2-D/3-D FEA

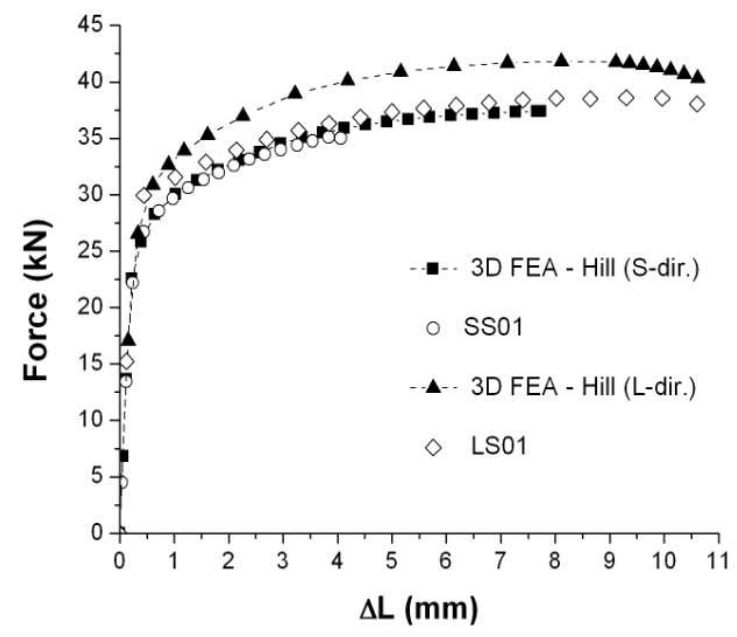

(a)

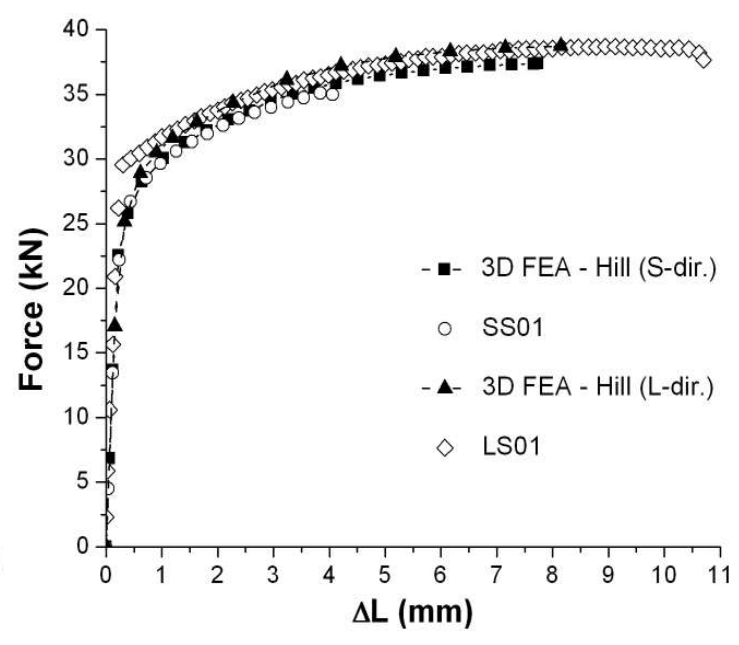

(b)

Figure 4. Force versus elongation in the S-directions using Hill's yield criterion; (a) FEA based on yield strengths and (b) ultimate strengths

strengths for the $S$-direction $\left(\sigma_{Y}^{S}=344 \mathrm{MPa}\right.$ and $\left.\sigma_{U T S}^{S}=439 \mathrm{MPa}\right)$ were approximately ten and three percent less than for the $L$-direction, or $\sigma_{Y}^{L}=395 \mathrm{MPa}$ and $\sigma_{U T S}^{L}=452 \mathrm{MPa}$, respectively. Hence, the finite element analysis for anisotropic behaviour is done either based on yield strengths or ultimate strengths for the $S$ - and $L$-directions. Based on yield strengths, the following six anisotropic coefficients for Hill's criterion, Eqn. $2 b$ were used, as given in Eqn. (5): 


$$
\begin{aligned}
& R_{11}=1.10, R_{22}=1.00, R_{33}=1.10 \\
& R_{12}=1.00, R_{13}=1.00, R_{23}=1.00 \\
& R_{i j}=\frac{\sigma_{i j}}{Q}, i, j=1,2,3 \\
& Q= \begin{cases}\sigma_{Y} & i=j \\
\tau_{Y} & i \neq j\end{cases}
\end{aligned}
$$

The given coefficients in Eqn. (5) are a clear indication of the presence of anisotropy along the $x$ and $z$ axes only, but not along the y axis, which is the loading direction in $S$-material orientation. It indicates that only planar anisotropy is present. Also, no anisotropic effect on shearing was considered $\left(R_{12}=1, R_{13}=1\right.$, $\left.R_{23}=1\right)$. The comparison between simulation and experimental results for this case is shown in Fig. 4(a).

It is evident from Fig. 4(a) that the Hill's criterion resulted in a close match with experimental results for the S-direction but overestimated the force at the onset of yield by approximately $7 \%$ for the $L$-direction. Based on ultimate tensile strengths in $S$ - and $L$-directions, the anisotropic coefficients for Hill's yield criterion are given in Eqn. (6):

$$
\begin{aligned}
& R_{11}=1.03, R_{22}=1.00, R_{33}=1.03 \\
& R_{12}=1.00, \quad R_{13}=1.00, R_{23}=1.00 \\
& R_{i j}=\frac{\sigma_{i j}}{Q}, i, j=1,2,3 \\
& Q= \begin{cases}\sigma_{Y} & i=j \\
\tau_{Y} & i \neq j\end{cases}
\end{aligned}
$$

It is obvious from Fig. 4(b) that the simulation results perfectly fit the experimental hardening behavior in the $L$-direction while underestimating the onset of yielding. It can be concluded that using Hill's yield criterion, both the onset of yielding and hardening behaviors in the $L$-direction cannot be accurately predicted. Therefore, the choice of anisotropic coefficients in Hill's yield criterion is mainly dependent on the application's need. For designing products involving plastic deformation, hardening behavior is more important as compared to the onset of yielding, while the reverse is true in the case of designing within the elastic limit. The results prove that there is no isotropic effect in shear for smooth bar.

\section{Conclusions}

Isotropic and anisotropic yield criteria were used to predict the yielding and hardening behaviors for $S$ - and $L$-directions in smooth bar using uniaxial tensile test specimens. Hardening behavior was determined only for $S$-direction. Three dimention finite element analyses for smooth bar were performed using isotropic and anisotropic yield criteria. Anisotropic parameters for Hill's criterion were determined using mechanical test data of smooth bar in the $L$-direction. Hill yield criterion slightly overestimated the hardening curve for the $S$-direction, while the effect was more pronounced in the $L$-direction. It can be concluded that by using Hill's yield criterion, yielding and hardening behaviors in the $L$-direction cannot be predicted precisely. Therefore, the selection of Hill's anisotropic coefficients is mainly dependent on application. The present work could be extended by including other anisotropic yield criteria to predict deformation behavior more accurately. Furthermore, the analysis of notched bar specimens could also be included to investigate the effects of notch radius on the anisotropy of materials.

\section{References}

Barlat F, Becker RC, Hayashida Y, Maeda Y, Yanagawa M, Chung K, Brem JC, Lege DJ, Matsui K, Murtha SJ, Hattori S (1997), Yielding description for solution strengthened aluminum alloys. International Journal of Plasticity 13:385401.

Barlat F, Lege DJ, Brem JC (1991), A six-component yield function for anisotropic materials. International Journal of Plasticity 7:693-712.

Bron F, Besson J ( 2004), A yield function for anisotropic materials: Application to aluminum alloys. International Journal of Plasticity 20(45):937-963.

Bron F, Besson J (2006), Simulation of the ductile tearing for two grades of 2024 aluminum alloy thin sheets. Engineering Fracture Mechanics 73(11):1531-1552.

Bron F, Besson J, Pineau A (2004), Ductile rupture in thin sheets of two grades of 2024 aluminum alloy. Materials Science and Engineering A 380(12):356-364.

Campo D, Williams C, Fillippov A, Cook L, Brisco D, Dean B, Ring L (2003), Monodiameter drilling liner - From Concept to Reality Paper SPE 79790, presented at the drilling conference held in Amsterdam, The Netherlands.

Hershey AV (1954), The plasticity of an isotropic aggregate of anisotropic face-centered cubic crystals. Journal of Applied Mechanics 21:241-249. 
Hill R (1954), The mathematical theory of plasticity. Clarendon Press, Oxford, UK.

Hosford WF (1972), A generalize isotropic yield criterion. Journal of Applied Mechanics 39:607-609.

Hosford WF (1996), On the crystallographic basis of yield criteria. Textures Microstructure 26-27:479493.

Karafillis AP, Boyce MC (1993), A general anisotropic yield criterion using bounds and a transformation weighting tensor. Journal of Mechanics of Physics and Solids 41:1859-1886.

Lam DF, Menzemer CC, Srivatsan TS (2010), A study to evaluate and understand the response of aluminum alloy 2026 subjected to tensile deformation. Materials and Design 31(1):166-175.

Lee HT, Shaue GH (1999), The thermomechanical behavior for aluminum alloy under uniaxial tensile loading. Materials Science and Engineering A 268(1-2):154-164.

Logan RW, Hosford WF (1980), Upper-bound anisotropic yield locus calculations assuming $<111>$ pencil glide. International Journal of Mechanical Sciences 22:419430.

Mimaroglu A, Yenihayat OF (2003), Modelling the superplastic deformation process of 2024 aluminium alloys under constant strain rate: Use of finite element technique. Materials and Design 24(3):189-195.

Nakai M, Eto T (2000), New aspect of development of high strength aluminum alloys for aerospace applications. Material Science and Engineering A 285(1-2):62-68.
Pervez T, Qamar SZ, Seibi AC, Al-Jahwari FK (2008), Use of SET in Cased and Open Holes: Comparison between Aluminum and Steel. Materials and Design 29:811-817.

Schmitt C, Lipinski P (1998), Modelling of the elasto-plastic behavior of rolled aluminium alloy for forming applications. Journal of Materials Processing Technology 74(1-3):14-22.

Shanmuga SN, Murugan N (2010), Tensile behavior of dissimilar friction stir welded joints of aluminium alloys. Materials and Design 31(9):41844193.

Srivatsan TS, Vasudevan S, Park L (2007), The tensile deformation and fracture behavior of friction stir welded aluminum alloy 2024. Materials Science and Engineering A 466(1-2):235-245.

Starke EA, Staley JT (1996), Application of modern aluminum alloys to aircraft. Progress in Aerospace Science 32(2-3):131-172.

Steglich D, Brocks W, Heerens J, Pardoen T (2008), Anisotropic ductile fracture of Al 2024 alloys. Engineering Fracture Mechanics 75:3692-3706.

Tresca H (1864), On the flow of solid bodies subjected to high pressures. In: Comptes-Rendus de l'Académie des Sciences Paris 59:754-756.

von Mises R (1913), Mechanik der festen Körper im plastisch- deformablen Zustand. Nachrichten von der Gesellschaft der Wissenschaften zu Göttingen, Mathematisch-Physikalische Klasse 582592:doi:citeulike-article-id:6897288. 